\title{
Adaptive Optics-Based Measurements of the Black Hole in Abell 2162-BCG
}

\author{
Nicholas J. McConnell ${ }^{1}$, James R. Graham ${ }^{1}$, Chung-Pei Ma ${ }^{1}$, \\ Karl Gebhardt ${ }^{2}$, and Tod R. Lauer ${ }^{3}$ \\ ${ }^{1}$ Astronomy Department, University of California, Berkeley, United States \\ Email: nmcc@berkeley.edu \\ ${ }^{2}$ Department of Astronomy, University of Texas, Austin, USA \\ ${ }^{3}$ National Optical Astronomy Observatory, USA
}

Keywords. galaxies: nuclei, galaxies: halos, galaxies: clusters: individual (Abell 2162)

We present preliminary measurements of the central black hole mass $M_{\mathrm{BH}}$, and stellar mass-tolight ratio $M_{\star} / L_{R}$, in the Brightest Cluster Galaxy of Abell 2162 (A2162-BCG), using integralfield unit (IFU) data from OSIRIS on Keck 2 with laser guide star adaptive optics (LGSAO). Our results demonstrate early success in an ongoing effort to obtain stellar dynamical measurements of $M_{\mathrm{BH}}$ in nine BCGs using ground-based AO.

Measuring $M_{\mathrm{BH}}$ in a statistically significant sample of BCGs will establish how $M_{\mathrm{BH}}$ scales with luminosity $L$ and velocity dispersion $\sigma$ in the most massive galaxies, indicating the number density of galaxies with $M_{\mathrm{BH}}>3 \times 10^{9} M_{\odot}$. At galaxy cluster scales, $M_{\mathrm{BH}}$ in the BCG sets the integrated energy from AGN feedback. The relationships between $M_{\mathrm{BH}}, L$, and $\sigma$ in the BCG also constrain the role of radial mergers in forming galaxy clusters.

Our measurements of $M_{\mathrm{BH}}$ and $M_{\star} / L_{R}$ in A2162-BCG use data from OSIRIS as well as seeing-limited, IFU data from GMOS on Gemini North. Out to $19 \mathrm{kpc}$, we adopt the major-axis kinematics of Carter, Bridges, \& Hau (1999). We use axisymmetric stellar orbit models, including a dark matter halo (Gebhardt \& Thomas 2009), to fit the observed line-of-sight kinematics. Because our data span insufficient radii to empirically distinguish enclosed halo mass from stellar mass, we have examined suites of models with four different fiducial halos. We measure lower values of $M_{\star} / L_{R}$ and higher values of $M_{\mathrm{BH}}$ as the enclosed halo mass increases (Figure 1). Our results reproduce the trend seen by Gebhardt \& Thomas (2009) for M 87, indicating that the extended dark matter profile of BCGs must be constrained to accurately determine $M_{\mathrm{BH}}$ from stellar dynamics.
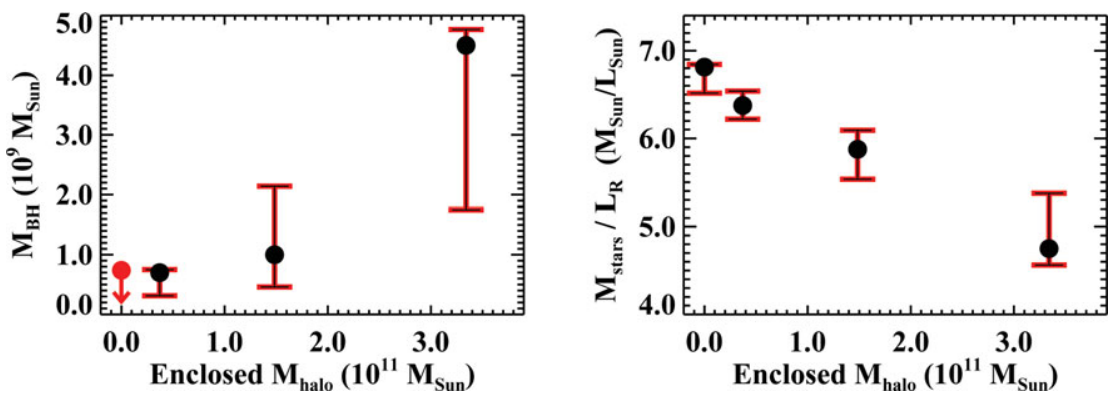

Figure 1. Measurements of $M_{\mathrm{BH}}$ and $M_{\star} / L_{R}$ in A2162-BCG, modeling four different dark matter halos. The enclosed halo mass is defined at $19 \mathrm{kpc}$. Error bars represent $68 \%$ confidence.

\section{References}

Carter, D., Bridges, T. J., \& Hau, G. K. T. 1999, MNRAS, 307, 131

Gebhardt, K. \& Thomas, J. 2009, ApJ, 700, 1690 